\title{
ON CONVOLUTION AND FOURIER SERIES
}

\author{
BY JACK BRYANT
}

Communicated by A. Zygmund, September 8, 1966

In [4, pp. 108-114], Salem found that each function in $L_{1}(0,2 \pi)$ (or $C[0,2 \pi]$ ) can be represented as the convolution of a function in $L$ (or $C$ ) with an even function in $L$ with convex Fourier coefficients. We announce here a slight generalization of this theorem, and some related results which follow from a study of our methods. Detailed proofs will appear elsewhere [2].

We require the following notation: If $f$ is a function, $\left(\tau_{h} f\right)(x)$ $=f(x+h) . B$ will denote a Banach space with norm $\|\cdot\|$. If $f \in L$, $S[f]$ denotes the Fourier series of $f,\left\{S_{n}\right\}$ the partial sums of $S[f]$ and $\left\{\sigma_{n}\right\}$ the $(C, 1)$ means of $S[f]$. $\|\cdot\|_{1}$ denotes the $L_{1}$-norm. If $\left\{\lambda_{n}\right\}$ is a sequence, $\Delta \lambda_{n}=\lambda_{n}-\lambda_{n+1}$ and $\Delta^{2} \lambda_{n}=\Delta \lambda_{n}-\Delta \lambda_{n+1}$. We define $Q$ to be the class of functions $g$ with $S[g]=\lambda_{0} / 2+\sum \lambda_{n} \cos n x$, where $\Delta^{2} \lambda_{n} \geqq 0$ and $\lambda_{n} \rightarrow 0$. Note each function in $Q$ is even, positive, integrable and differentiable on $(0, \pi) . A$ will denote an absolute constant, not necessarily the same each time it appears.

Theorem 1. Suppose $S=\sum A_{n}$ is summable $(C, 1)$ to $f$ in a Banach space $B$. Let $\phi$ be a positive increasing function with $\int_{0}^{\infty} 1 / \phi(t) d t<\infty$. Let $\left\{\sigma_{n}\right\}$ be the $(C, 1)$ means of $S$; if $\left\{\lambda_{n}\right\}$ is a sequence such that $0<\lambda_{n} \leqq \phi^{-1}\left(\left\|\sigma_{n}-f\right\|^{-1}\right), \Delta^{2} \lambda_{n} \leqq 0$ and $\lambda_{n} \uparrow \infty$, then the series $T=\sum \lambda_{n} A_{n}$ is summable $(C, 1)$ in $B$.

Theorem 2. Let $B C L$ be a Banach space with $\|u\|_{1} \leqq A\|u\|$ for each $u$ in $B$, and suppose the $(C, 1)$ means of $S[f]$ are in $B$ and $\left\|\sigma_{n}-f\right\| \rightarrow 0$. Then there exists $g \in Q$ and $h \in B$ such that $f=g * h$.

Theorem 3. Let $f \in L$. Then $f=g * h$, where $g \in Q$ and $S[h]$ and $S[f]$ have, except for a set of measure zero, the same points of convergence.

Theorem 4. Suppose $f \in L$, and let $\left\{\sigma_{n}\right\}$ be the $(C, 1)$ means of $S[f]$. If $\sum\left\|\sigma_{k}-f\right\|_{1} / k<\infty$ and if $\left\|\sigma_{k}-f\right\|_{1}=o(1 / \log k)$, then $S[f]$ converges almost everywhere.

If we suppose more about $B$, Theorem 2 can be completed as follows:

THEOREM 5. Let BCL satisfy the following conditions: $B$ is a Banach space and

(1) for each $u$ in $B,\|u\|_{1} \leqq A\|u\|$,

(2) for each $u$ in $B$ and each $h,\left\|\tau_{h} u\right\| \leqq A\|u\|$, 
(3) for each $f$ in $L([0,2 \pi) \times[0,2 \pi))$,

$$
\left\|\int_{0}^{2 \pi} f(\cdot, t) d t\right\| \leqq A \int_{0}^{2 \pi}\|f(\cdot, t)\| d t
$$

(4) the partial sums of $S[u]$ are in $B$ when $u$ is in $B$.

Then the following four conditions on an element $f$ of $B$ are equivalent:

(i) $\omega_{1}(f ; t)=\sup \left\{\left\|\tau_{h} f-f\right\|: 0 \leqq|h| \leqq t\right\}=o(1)$,

(ii) $\omega_{2}(f ; t)=\sup \left\{\left\|\tau_{h} f+\tau_{-h} f-2 f\right\|: 0 \leqq|h| \leqq t\right\}=o(1)$,

(iii) $S[f]$ is summable $(C, 1)$ to $f$ in $B$,

(iv) $f=g * h, \quad h \in B$ and $g \in Q$.

Indication of Proofs. Theorem 1 is proved by summing the expression for the $(C, 1)$ means $\tau_{n}$ of $T=\sum \lambda_{n} A_{n}$ by parts twice and showing the sequence $\left\{\tau_{n}\right\}$ is Cauchy in $B$. We use Theorem 1 to obtain Theorem 2; the hypothesis $\|u\|_{1} \leqq A\|u\|$ is necessary to show the series constructed is $S[h]$ for $h \in B$. To prove Theorem 3, we construct a seminorm on $L$ so that convergence with respect to this seminorm implies a.e. convergence in the set of points of convergence of $S[f]$. We then use machinery developed for Theorem 1 plus the fact $\left\|\sigma_{n}-f\right\|_{1} \rightarrow 0$ to obtain Theorem 3. Theorem 4 is an observation based on the fact that the numbers $\{1 / \log n\}$ are convergence factors for Fourier series. (We note that in Theorem $4, \sigma_{n}$ may be replaced by $s_{n}$ each time it appears.) Theorem 5 requires hypothesis (3), (4) for (ii) $\rightarrow$ (iii), (1) for (iii) $\rightarrow$ (iv) and (2) for (iv) $\rightarrow$ (i); (iii) $\rightarrow$ (iv) is Theorem 2, and the other proofs are elementary.

\section{REFERENCES}

1. Jack Bryant, Theorems relating convolution and Fourier series, Ph.D. Thesis, Rice University, Houston, Texas, 1965.

2. - On convolution and Fourier series, Duke Math J. (to appear).

3. - On convolution and moduli of continuity, (to appear).

4. R. Salem, Sur les transformations des series de Fourier, Fund. Math. 33 (1939) 108-114.

Texas A\&M University 